\title{
Head movement and allomorphy in children's negative questions
}

\author{
Marjorie Pak ${ }^{*}$
}

\begin{abstract}
English-speaking preschoolers occasionally produce negative questions with a 'doubled' auxiliary (e.g. Why did you didn't know?). These 2AUXQs apparently involve a failure to raise [NEG $n$ 't] to C (cf. Why didn't you know?). I analyze $2 A u x Q s$ as the product of two independent errors: a planning error (raising T-to-C without raising Neg-to-T first) and an allomorphy error (overgeneralization of $-n$ 't). The planning error results from lack of practice: serial head-movement is relatively uncommon in English, and true Neg-to-T-to-C may be rarer than appearances suggest. In e.g. Why don't we play, ok?, -n't is not interpreted within $\mathrm{TP}$ - and strikingly, 2AuxQs are unattested here.
\end{abstract}

Keywords. syntax of questions; head-movement; allomorphy; acquisition

1. Introduction. Many English-speaking children, once they are regularly using subject-AUX inversion, produce occasional errors like (1): negative $n$ 't questions with a 'doubled' auxiliary (henceforth 2AUXQs):

(1) a. Why does Superman doesn't wear Underoos on his bottom? $(3 ; 03)$

(MacWhinney 2000)

b. Why did you didn't know? $(3 ; 08)$ (Kuczaj 1977; abe126)

c. What do we don't have that we can make? (3;09) (Kuczaj 1977; abe135)

Compared to the adult questions in (2), 2AuxQs seem to involve a failure to raise $n$ ' $t$ to $\mathrm{C}$, so that $n$ ' $t$ gets stranded and eventually rescued by a copy of the auxiliary (3). See Guasti et al. (1995), Hiramatsu (2003), Xu \& Snyder (2011), and Zuckerman (2001), for precedent for this basic idea.

(2) a. Why doesn't Superman wear Underoos on his bottom?

b. Why didn't you know?

c. What don't we have that we can make?

a. Neg-to-C raising: Why didn't [те you $\boldsymbol{t}$ know]? b. Neg-to-C failure $(2 A u x Q)$ :

Why did [TP you didn't know]?

But why does Neg-to-C fail? Previous accounts disagree here, and none are quite satisfactory: they either fail to explain important facts, or rely on unmotivated assumptions about differences in the child's grammar (see $\S 2$ ). This paper aims to fill this gap.

In $\S 3$, I look at a range of negative questions in CHILDES (MacWhinney 2000) and show that:

- 2 AuxQs never occur in tag questions, positive-bias yn-questions, or why - n't proposals, but only in information-seeking questions with 'true' sentential negationsupporting the idea that Neg-to-C failure is at work here.

\footnotetext{
* Thanks to Anna Bintinger, Stephanie Long, Doris Zhou, and my LSA reviewers and audience for their helpful comments and suggestions. All errors are my own. Author: Marjorie Pak, Emory University (mgpak@emory.edu).
} 
- On the other hand, 2AuxQs do occur in inner-negation $y n$-questions, where Neg-to-C is not even expected (e.g. Do we not really need it? $\neq$ Don't we really need it?)suggesting that Neg-to-C failure is only part of the story.

Building on these findings, I argue in $\S 4$ that $2 A u x Q s$ involve two independent errors, both well-precedented: (i) a planning error (raising $\mathrm{T}$ to $\mathrm{C}$ without raising Neg to $\mathrm{T}$ first), and (ii) an allomorphy error (overgeneralization of $n^{\prime} t$ (vs. not)).

\section{Previous approaches to $2 \mathrm{AuxQs}$}

2.1. EXPLAINING NEG-TO-C FAILURE. While 2 AuxQs are rare in spontaneous-speech corpora (Stromswold 1990), Guasti, Thornton \& Wexler (1995, henceforth GTW) show that children do produce them in appropriate contexts, e.g.:

(4) Puppet: I heard the snail doesn't like to eat some things. Ask him what.

Target: What don't you like to eat?

$2 A u x Q$ : What do you don't like to eat?

All 10 of GTW's participants $(3 ; 08-4 ; 07)$ produced at least some 2 AuxQs in such contexts, five of them $\geq 45 \%$ of the time.

GTW attribute their findings to a general syntactic ban on raising Neg out of TP (cf. (3b)). This ban is initially adopted as a UG option, and continues to hold in languages like Paduan, but is overridden in English in the face of counterevidence in the adult input, viz. questions like (2).

Hiramatsu (2003), however, raises a serious problem for GTW: a mismatch between children's grammaticality judgments and what they produce. Three of Hiramatu's four participants $(4 ; 0-5 ; 0)$ who produced 2 AuxQs $>80 \%$ of the time rejected 2 AuxQs like $(5 \mathrm{a}) \geq 75 \%$ of the time in a judgment task. On the other hand, they overwhelmingly accepted adultlike AUXn't-initial questions like (5b) - which would be unexpected if they were using a grammar that banned Neg-to-C.

a. Why did Bert didn't cook the eggs? (rejected)

b. Why didn't Big Bird brush the dog? (accepted)

This production/judgment mismatch also poses a problem for Zuckerman (2001:ch8), who attributes Neg-to-C failure to children's preference for 'more economical' derivations.

Zuckerman's idea is that when children are exposed to two structures that are nearly identical in meaning (in this case, Neg-to-C and Neg in situ), they 'reject the possibility that both alternatives are allowed' (p. 158) and prefer the derivation with less movement. Again, as with GTW, Negto-C failure is a direct product of the child's nonadultlike grammar, and so there is no explanation for Hiramatsu's (2003) production/judgment mismatch.

Given that Neg-to-C failure appears to be production-specific, Xu \& Snyder (2011) characterize 2 AuxQs as performance errors, an idea I am sympathetic to (see §4). But the particular type of error they posit - misanalyzing $n$ 't as a specifier of NegP, so that it is ineligible for head-movement-is to my knowledge unprecedented. There is no independent evidence for specifier-head confusion, as they acknowledge.

In a similar vein, Hiramatsu (2003) proposes that 2 AuxQs have constituent negation, with $\mathrm{Neg}$ as a vP adjunct-rendering Neg-to-C head-movement structurally impossible. But again, the assumption that these children merge Neg $n$ 't in a different structural position from adults is unmotivated. There is no independent reason why children this age would overuse constituent 
negation - particularly in contexts like (4) where they they have just heard a puppet modeling normal sentential negation (with $d o$-support).

Indeed, children this age generally have adultlike negation: when GTW elicited negative declaratives, children produced adultlike forms 'without exception' (p. 231); they did not produce e.g. (6)a, which might be expected if they customarily treated Neg as a vP adjunct. It is also worth noting that children this age do not (to my knowledge) produce overregularized contractions like (6)b in 2AuxQs, as we might expect if $n$ ' $t$ were simply 'leaning' phonologically on its host rather than fully incorporating via head-movement.
a. *You [vp not [vP like pizza]]
b. *Why do you $[d u] n$ 't like pizza?

All four of the proposals reviewed so far (GTW, Zuckerman 2001, Hiramatsu 2003, and Xu \& Snyder 2011) agree that there is something problematic about raising $n^{\prime} t$ to $\mathrm{C}$ at this stage in acquisition. The goal here is to maintain this basic assumption but also explain why 2AuxQs are production-specific, without stipulating unmotivated differences in the child's grammar.

2.2. OVERUSE OF THE CONTRACTED FORM $N^{\prime} T$. As the reader may have noticed, there is a way to rescue a derivation in which Neg-to-C (for whatever reason) fails, without sacrificing grammaticality. Namely, if Neg is left behind in TP but then spelled out as the uncontracted form not, the result is grammatical (albeit relatively infrequent in colloquial speech; see Pak 2017).

a. Why did [те you not know]?

b. What do [тр you not like to eat]?

GTW show that children who produce 2 AuxQs also sometimes produce questions like (7). The question that arises, then, is why children ever produce $2 A u x Q s$ instead of questions like (7).

As recognized by Zuckerman (2001:159), 'the explanation seems to be related to the children's preference for the contracted form of negation.' In other words, children show an overpreference for $n^{\prime} t$ (vs. not) — and, importantly, this overpreference is an independent problem from the Neg-to-C problem discussed in the previous subsection. This generalization plays an important role in my proposal in $\S 4$.

(8) Generalization: 2AuxQs involve the co-occurrence of two independent problems:

(i) a Neg-to-C raising problem and (ii) an overpreference for $n$ ' $t$ (vis-à-vis not).

3. New facts. I now turn to two observations, supported by CHILDES data (MacWhinney 2000), which have been overlooked in previous work.

3.1. OBSERVATION 1. First, 2AuxQ errors are unattested in tag questions, positive-bias $y n$ questions, and why - $n$ 't proposals (9) - a surprising gap given how frequent these utterances are in discourse. Table 1 shows how various negative-question types are distributed in the Kuczaj (1977) corpus of naturally occurring speech.

a. Positive-bias yn-questions: Ow, doesn't that hurt?

Unattested: Ow, does that doesn't hurt?

b. Tag questions: A tricycle has a back, doesn't it?

Unattested: A tricycle has a back, does it doesn't?

c. Why-n't proposals: Why don't we play a game, ok?

Unattested: Why do we don't play a game, ok? 


$\begin{array}{lcc} & \frac{n(\%)}{2 \text { Aux }} \\ \text { AUXn't-initial yn-questions } & 88(52 \%) & -- \\ \text { Tag questions } & 26(15 \%) & 0 \\ \text { Why-don't proposals } & 7-22^{1}(4-13 \%) & 0 \\ \text { Other (e.g. (1)b,c) } & \frac{34-49(20-28 \%)}{170(100 \%)} & 5\end{array}$

Table 1: Abe's inverted negative questions (2;6-5;0) (data from Kuczaj 1977)

Holmberg (2016:ch4.8) suggests that $n$ 't in (9)a,b is not actually merged TP-internally, but rather heads a functional projection above TP, in the C-domain. Among other things, this would explain why (9)a,b cannot be answered in a way that 'confirms' TP-internal negation (10):

a. Doesn't that hurt? A: Yes, you're right, it \{does / *doesn't $\}$.

b. A tricycle has a back, doesn't it? A: That's right, it \{does / *doesn't .

It is quite plausible that $n$ 't in (9)c, too, is interpreted outside of TP. Negative why questions normally entail an entire negative TP (11)a, but why - n't proposals do not (11)b. In fact, the supposedly reconstructed negative TP in a why $-n$ 't proposal is sometimes not even fully grammatical (11)c.

(11) a. Why don't cats like chocolate?

[CP why NEGi [TP cats NEGi like chocolate]] ( $\Rightarrow$ Cats don't like chocolate.)

b. Why don't we play a game, ok?

[CP why-NEG [TP we play a game]] ( $\nRightarrow$ We don’t play a game.)

c. Why don't you stop that right now?

[CP why-NEG [TP you stop that right now ]] ( $\nRightarrow$ ??You don't stop that right now.)

Overall, Observation 1 further bolsters the already widely accepted idea that 2AuxQs are at least partly attributable to a problem with Neg-to-C raising. The fact that children have no trouble producing $n$ ' $t$ in $\mathrm{C}$ in (9) can be taken as a new kind of evidence that the $n$ ' $t$ in these questions does not originate within TP.

3.2. OBSERVATION 2. Interestingly, some 2AuxQs occur in contexts where Neg is not even expected to raise out of TP in the first place - namely, 'inner-negation' $y n$-questions, where negation has TP-internal scope. While GTW seem to assume that Neg-to-C is the target in negative $y n$-questions as well as negative $w h$-questions, many English speakers avoid raising Neg to $\mathrm{C}$ in inner-negation $y n$-questions, instead leaving Neg in situ spelled out as not (Sailor 2003, pace Ladd 1981).

a. I don't like him; do you not like him either?

b. I don't like him; ${ }^{\%}$ don't you like him either?

\footnotetext{
${ }^{1}$ Fifteen of Abe's why-don't questions are ambiguous between information-seeking and proposal readings. Diagnostics for distinguishing these two types include the okay? tag (only available with proposals), NPIs and PPIs (e.g. Why don't we ever go to the movies? can only be information-seeking), the presence of stative or non-agentive verbs (e.g. Why aren't you a teacher? can only be information-seeking), and the use of simple-present tense alongside punctual adverbs (Why don't we eat now? can only be a proposal). When none of these diagnostics are present (e.g. Why don't we play in the kitchen?), the question is ambiguous between an information-seeking reading and a proposal reading.
} 
Nevertheless, 2Aux errors are attested in inner-negation yn-questions. In (13), for example, Abe $(3 ; 06)$ has just said that he didn't like the spooky parts of the show and is now asking his father if he also disliked them.

(13) FAT: did you like that show we went to see?

CHI: $\quad($ ex)cept $<$ I didn't $>$ I didn't like the spooky parts.

FAT: which parts were spooky?

CHI: the cougar part and the cougar part.

FAT: those were spooky parts huh?

CHI: did you don't like the spooky parts?

FAT: I don't like spooky parts.

CHI: did you when you were a little kid?

FAT: what?

CHI: did you when you were a little kid?

FAT: did I like (th)em then?

CHI: uhhuh.

FAT: nope

An adultlike counterpart to Abe's 2AuxQ here would be:

Did [тр you not like the spooky parts (either)]?

cf. ${ }^{\%}$ Didn't you like the spooky parts either?

Here are some more inner-negation $y n$-2AuxQs I observed in my own child's speech $(5 ; 0-5 ; 1)$. The contexts are included to show that these questions are negative-biased, rather than positivebiased, and thus correspond to the adult questions in (16) with TP-internal not rather than to their counterparts with $n$ 't in C.

(15) a. Do we don't need to lock it [the car]? (already walking away from car)

b. Do I don't need to wear socks? (already putting on shoes over bare feet)

c. Do we don't really need our little things [headlights] on? (on a sunny day)

d. Do they [cats] normally don't drink? (previously was told that cats don't drink much)

Again, the adult counterparts to these $2 \mathrm{AuxQs}$ - in these contexts - would not have Neg-to-C:

a. Do we not need to lock the car?

cf. "Don't we need to lock the car? (already walking away from car)

b. Do I not need to wear socks?

$c f$. "Don't I need to wear socks? (already putting on shoes over bare feet)

c. Do we not really need our headlights on?

$c f$. "Don't we really need our headlights on? (on a sunny day)

d. Do they normally not drink?

cf. "Don't they normally drink? (already knows that they normally don't drink much

The fact that 2Aux errors occur in inner-negation $y n$-questions shows that 2AuxQs cannot be solely attributed to Neg-to-C failure; rather, there must be an additional problem at work. As suggested in (8), I believe this additional problem is children's overpreference for $n$ ' $t$ (as opposed to not). The proposal is laid out in detail next.

4. Analysis. I propose that $2 \mathrm{AuxQ}$ s involve two independent errors: (i) a planning error (raising $\mathrm{T}$ to $\mathrm{C}$ without raising Neg to T first), and (ii) an allomorphy error (overgeneralization of $n$ 't (vis-à-vis not)). 
Let's begin with the syntax I am assuming for two types of adult negative questions - $\mathrm{a}$ negative why question with $n^{\prime} t$ (17)a and an inner-negation $y n$-question with not (17)b:

a. Negative why question

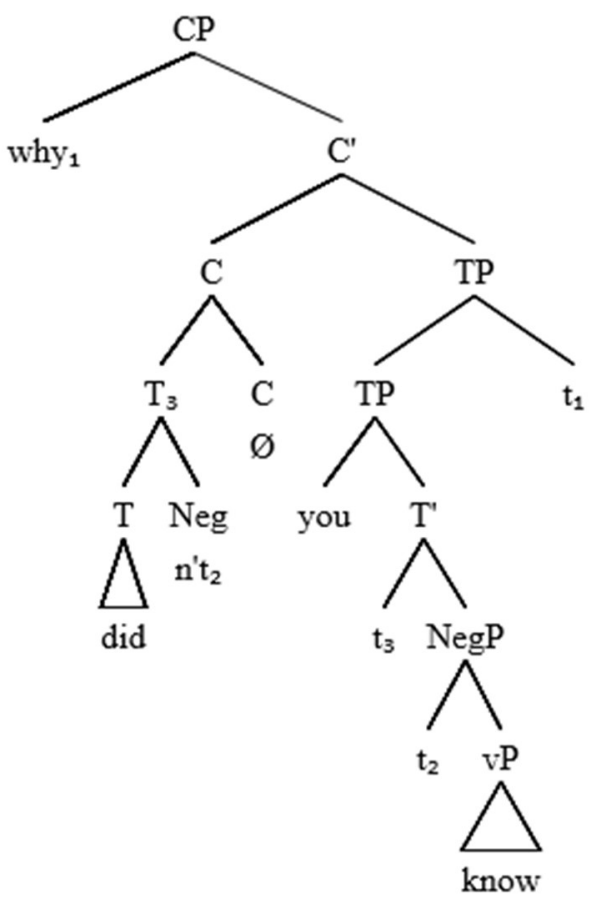

b. Inner-negation $y n$-question

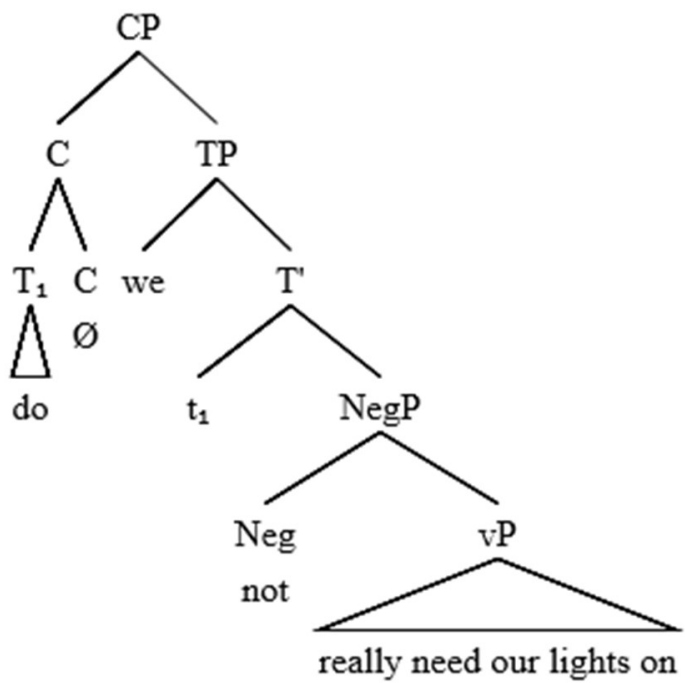

I have adopted the following assumptions here (among others):

- $\quad \mathrm{NegP}$ is a functional projection between TP and vP;

- $\quad n^{\prime} t$ and not are allomorphs of Neg (see (18));

- Neg ordinarily raises to T in (17)a but stays in situ in (17)b; ${ }^{2}$

- T obligatorily raises to $\mathrm{C}$ in English direct questions (yielding subject-AUX inversion);

- When bound $\mathrm{T}$ is prevented from combining with $v$, dummy verb $d o$ is inserted.

Adult allomorphy rule for Neg:

[+NEG] $\leftrightarrow$ n't / $X \oplus_{\text {_ }}$ ('iff Neg is affixed to $\mathrm{X}$, where $\mathrm{X}$ is phonologically overt') not (elsewhere; the default)

Now let's consider what happens in a 2AuxQ. Given the production/judgment mismatch revealed by Hiramatsu (2003), I agree with Xu \& Snyder (2011) that (some) 2AuxQs are attributable to a performance-related problem. But I appeal to a more general type of error than theirs - namely, a planning error.

Speakers sometimes begin uttering sentences before they are fully planned (see Bock 1995 and references cited there). Suppose that in the 2AuxQ Why did you didn't know? (3b), the child begins the utterance with Why did..., raising T-to-C, without planning far enough ahead to realize that Neg needs to be raised to $\mathrm{T}$ first. Neg is then stranded within TP.

\footnotetext{
${ }^{2}$ A full account of why Neg-to-C is dispreferred in inner-negation $y n$-questions is beyond the scope of this paper. One possibility, building off Romero \& Han (2004), might be that raising Neg to C would allow Neg to scope over a VERUM operator in C, yielding an undesired reading. See e.g. Pak (2017).
} 
Utterance begins: $\quad\left[\right.$ СР why $\left[\mathrm{C}\left[\mathrm{T} \operatorname{did}_{\mathrm{i}}\right]\right][\mathrm{TP} \ldots$

Utterance continues: ... [TP you $t_{\mathrm{i}}[\mathrm{NegP}$ !Neg ...

A possible parallel error in adult speech might be possessor-extraction utterances with resumptive pronouns:

a. *John is the guy who I once met his mother.

(cf. John is the guy whose mother I once met.)

b. Utterance begins: John is the guy $\left[\mathrm{CP} \mathrm{who}_{\mathrm{i}} . .\right.$.

Utterance continues: ...[TP I once met [DP $t_{\mathrm{i}}$ !mother ]]]

As with children's 2AuxQs, the idea is that the speaker begins the utterance without having planned far enough ahead to 'see' something that should have moved forward (in this case, mother should have been pied-piped). Kroch (1981) proposes a similar analysis of resumptive pronouns in questions with island violations (e.g. the guy who I hate almost everything he does), using a real-time sentence generator with limited capacity for advance planning.

Why would Neg-to-C questions be difficult for children to plan? We saw in $\S 3.1$ that 'true' negative questions - i.e. questions where $n$ ' $t$ surfaces in $\mathrm{C}$ but is unambiguously interpreted within $\mathrm{TP}$ - are less common in discourse than they appear at first sight (Table 1). Moreover, Neg to $\mathrm{C}$ requires iterative head-movement - Neg to $\mathrm{T}$, then $\mathrm{T}$ to $\mathrm{C}$ - which may well stymie a child who has only recently mastered subject-AUX inversion (T-to-C).

As noted in $\$ 2.2$, the derivation in (19) could be 'rescued' by the allomorphy rule (18), which would insert not:

$$
\begin{array}{ll}
\text { Utterance begins: } & {\left[\mathrm{CP} \text { why }\left[\mathrm{C}\left[\mathrm{T}_{\mathrm{T}} \operatorname{did}_{\mathrm{i}}\right]\right][\mathrm{TP} . . .\right.} \\
\text { Utterance continues: } & \ldots \text { [т you } t_{\mathrm{i}}[\mathrm{NegP} \text { not know }
\end{array}
$$

This is where the second error becomes apparent. As is well known, English-speaking preschoolers are still in the process of acquiring allomorphy rules for [PAST], [PL], [DEF], etc. (Yang 2016); here are some overgeneralization errors produced by Abe at 3;08-3;09.

$$
\text { a. Mommy throwed the card away. (Kuczaj 1977, abe 126) }
$$

b. We're gonna go to Texas and fly $a$ airplane. (Kuczaj 1977, abe135)

The overuse of $n$ ' $t$ in 2AuxQs can also be analyzed as overgeneralization-mistakenly treating $n$ 't as the default and not as the restricted allomorph, to be inserted iff Neg has stress or focus (e.g. I do nót like him).

$$
\begin{aligned}
& \text { Children's allomorphy rule for Neg: } \\
& {[+\mathrm{NEG}] \leftrightarrow \text { not / } \overline{\text { n't (elsewhere; the default) }}}
\end{aligned}
$$

Children plausibly start with $n$ ' $t$ as the default because it is the more frequently occurring form in discourse (similar to $a$ in (22)b; see Pak 2016). If a child with the allomorphy rule in (23) derives (19) above, they will insert their default $n$ 't at Neg, a context where it has no host. As a last resort, the child either pronounces the lower copy of [ ${ }_{\mathrm{T}}$ does] or inserts an(other) instance of dummy do to support $n$ 't.

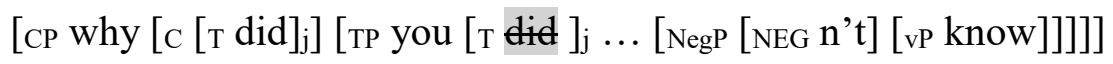

5. Predictions. The current account, unlike GTW, explains why children who produce 2 AuxQs overwhelmingly accept adultlike questions like Why didn't Big Bird brush the dog? (Hiramatsu 
2003). Under my proposal, Neg-to-C is fully grammatical for children, but difficult for some children to execute in production.

An important feature of the current analysis is that the planning error (i) and the allomorphy error (ii) are orthogonal to each other. Their independence, schematized in Table 2, leads to a number of predictions. Rather than having only two options - fully adultlike (top-left cell) and fully nonadultlike (bottom-right cell) - it is possible for individual children to master planning before allomorphy, or vice versa.

For example, it is possible for a child to still struggle with Neg-to-C planning (i) after they have acquired the adult allomorphy rule (ii); this pattern will yield e.g. Why did you not know? (21). Evidence for this pattern (represented in the top-right cell in Table 2) is given in GTW, where 7 of 10 participants produced questions like (21) alongside 2 AuxQs, two of them $>20 \%$ of the time. Questions like (21), while grammatical, are nonadultlike, since adults overwhelmingly use $n$ 't in negative why questions (Pak 2017).

Conversely, it is possible for a child to have adultlike Neg-to-C planning (i) but not yet have adultlike allomorphy (ii). As we saw, this pattern explains why 2 AuxQs are attested in inner-negation $y n$-questions, where Neg-to-C is not even the target (bottom-left cell in Table 2).

\begin{tabular}{cll} 
& Planning: + & Planning: - \\
\hline $\begin{array}{c}\text { Allomorphy: } \\
+\end{array}$ & Why didn't you know? (17)a & Why did you not know? (17)a \\
& Do we not really need them? (17)b & Do we not really need them? (17)b \\
\hline $\begin{array}{c}\text { Allomorphy: } \\
-\end{array}$ & Why didn't you know? (17)a & Why did you didn't know? (17)a \\
& Do we don't really need them? (17)b & Do we don't really need them? (17)b \\
\hline
\end{tabular}

Table 2: Four possible patterns in the development of negative questions

The absence of 2AuxQs in tag questions, positive-bias $y n$-questions and why -n't proposals ( $\$ 3$, Observation 1) lends independent support to the idea that $n$ ' $t$ here is not 'normal' sentential negation but rather a functional head merged in the C domain (Holmberg 2016). Since this $n$ ' $t$ is merged above TP, it gets picked up in the course of T-to-C raising without the degree of forward-planning necessary in e.g. (17)a.

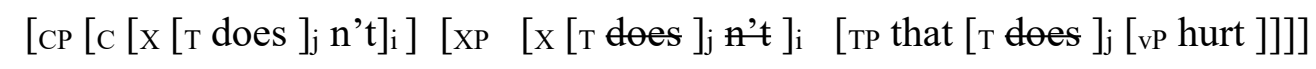

6. Conclusions. Under my treatment, $2 A u x Q s$ arise from the confluence of well-established properties of English morphosyntax: the status of NegP, the $n$ 't $\sim$ not alternation and affixhood of $n^{\prime} t$, and the restrictedness of serial head-movement, particularly Neg-to-C. The two errors that are held responsible for 2AuxQs - planning and allomorphy - are both well-established, and this account correctly predicts that children may struggle with one, both, or neither type of error.

Taken together, Observations 1 and 2 (\$3) show that 'true' negative questions with $n$ 't in C are less common than they appear at first sight. Many questions with $n$ 't in C turn out not to have true sentential negation (Observation 1; (25)), and some questions with true sentential negation do not allow Neg-to-C (Observation 2; (17)b). This finding may have implications for English syntax beyond acquisition, perhaps shedding light on the (somewhat mysterious) dispreference for Neg-to-C in e.g.:

(26) a. How do you not love this movie?! (??How don't you love this movie?!) (Pak 2017)

b. No way does he not like you. (" No way doesn't he like you.)

c. Had I not seen you, I wouldn't have stopped. (*Hadn't I...) (Zuckerman 2001:30) 


\section{References}

Bock, K. 1995. Sentence production: From mind to mouth. In Joanne Miller \& Peter Eimas (eds.), Handbook of perception and cognition, Vol. 11 : Speech, language, and communication. 181-216. Orlando, FL: Academic Press

Guasti, Maria Teresa, Rosalind Thornton \& Kenneth Wexler. 1995. Negation in children's questions: The case of English. Proceedings of BUCLD 19. 228-239.

Hiramatsu, Kazuko. 2003. Children's judgments of negative questions. Language Acquisition 11. 99-126. https://doi.org/10.1207/s15327817la1102 2.

Holmberg, Anders. 2016. The syntax of yes and no. Oxford: Oxford University Press.

Kroch, Anthony. 1981. On the role of resumptive pronouns in amnestying island constraint violations. Papers from the 17th Regional Meeting of CLS, 125-135.

Kuczaj, Stan A. 1977. The acquisition of regular and irregular past tense forms. Journal of Verbal Learning and Verbal Behavior 16. 589-600. https://doi.org/10.1016/S00225371(77)80021-2.

Ladd, D. Robert. 1981. A first look at the semantics and pragmatics of negative questions and tag questions. Papers from the $17^{\text {th }}$ Regional Meeting of CLS. 164-171.

MacWhinney, Brian. 2000. The CHILDES Project: Tools for analyzing talk. Lawrence Erlbaum.

Pak, Marjorie. 2016. How allomorphic is English article allomorphy? Glossa 1(1): 20. 1-27. http://doi.org/10.5334/gjgl.62.

Pak, Marjorie. 2017. Propositional how questions and negation. Proceedings of the 34th West Coast Conference on Formal Linguistics. 423-430. Somerville, MA: Cascadilla Proceedings Project. http://www.lingref.com/cpp/wccfl/34/paper3348.pdf.

Romero, Maribel \& Chung-hye Han. 2004. On negative yes/no questions. Linguistics and Philosophy 27. 609-658. https://doi.org/10.1023/B:LING.0000033850.15705.94.

Sailor, Craig. 2013. Questionable negation. Handout from paper presented at LSA Annual Meeting. Retrieved from www.craigsailor.net/papers/LSA 2013 handout.pdf.

Stromswold, Karin J. 1990. Learnability and the acquisition of auxiliaries. Cambridge, MA: MIT dissertation.

$\mathrm{Xu}$, Ting \& William Snyder. 2011. Children's 2Aux negative questions: Elicited production versus spontaneous speech. Proceedings of GALANA 2010. 277-285. http://www.lingref.com/ cpp/galana/4/paper2602.pdf.

Yang, Charles. 2016. The price of linguistic productivity: How children learn to break the rules of language. Cambridge, MA: MIT Press.

Zuckerman, Shalom. 2001. The acquisition of 'optional' movement. Groningen: University of Groningen dissertation. 